PharmacoEconomics \& Outcomes News 882, p2 - 10 Jul 2021

\title{
Best COVID-19 vaccine allocation strategy using a net-monetary benefit approach?
}

Researchers from Canada and the UK have found that optimal COVID-19 vaccination allocation is complex and depends on the primary outcome that has been prioritised and which public health policies are in place - outcomes appear best when supported by intervention reducing contact in higher-risk patient sub-groups.

They developed an transmission-dynamic epidemiological model (the COVID-19 Risk Assessment Model [CRAM]) stratified by age groups (up to $75+$ years), risk (high-risk and not high-risk) and calibrated to the time-variant state of public health interventions and human behaviour using data from Alberta, Canada. The model had 5 compartments: susceptible; exposed; infected; isolation; and, recovered. The model was used to calculate active cases, hospitalisations, total cost*, quality-adjusted life years and net monetary benefit (NMB) for a range of hypothetical vaccination strategies (versus no vaccine) that included different vaccine efficacies and coverage.

For a scenario where there is $40 \%$ coverage with $80 \%$ vaccine efficacy and prioritisation of people 60 years at high risk of poor outcomes, there was a 17\% reduction in active cases and the NMB increased by \$Can263 million, compared with no vaccination. When no prioritisation of age or risk groups was in place, non-pharmaceutical interventions such as school closures and contact restriction of older citizens had similar impact on the incremental NMB (\$352 vs \$292 million, respectively). However, if older age groups were given vaccination priority, the relative benefit of school closures was much greater ( $\$ 214$ vs $\$ 118$ million). The cumulative cases and incremental NMB varied substantially depending on which concurrent strategies were in place, and the optimal vaccination strategy was sensitive to whether policies targeted morbidity reductions or NMB outcomes.

The authors note that "in our model, NMB is improved relative to baseline when seniors continue to reduce their number of contacts, and if vaccines are allocated without prioritising older and high-risk population groups. However, this would mean that the people with the greatest risk of disease and poor outcomes would also bear the highest burden through additional social isolation."

* Canadian health payer system perspective and presented in 2020 Canadian dollars

Kirwin E, et al. A Net Benefit Approach for the Optimal Allocation of a COVID-19 Vaccine. PharmacoEconomics : 17 Jun 2021. Available from: URL: http:// doi.org/10.1007/s40273-021-01037-2 